\section{Sobrevida de cinco anos e fatores associados ao câncer de boca para pacientes em tratamento oncológico ambulatorial pelo Sistema Único de Saúde, Brasil}

\author{
Specific 5-year oral cancer survival and associated \\ factors in cancer outpatients in the Brazilian \\ Unified National Health System
}

\section{La supervivencia a cinco años para pacientes con cáncer oral en radioterapia o quimioterapia en el Sistema Único de Salud, Brasil}

\begin{abstract}
This study aimed to analyze the specific five-year oral cancer survival rate and associated factors in Brazil. This was a retrospective cohort study using the Oncological Database as the source, with probabilistic-deterministic linkage of all the records for authorization of radiotherapy and/or chemotherapy in the Unified National Health System from 2000 to 2006, generating a single record for each patient. The current study included patients diagnosed with oral cancer from 2002 to 2003 (except for cancer of lip), and ranging from 19 to 100 years of age $(N=6,180)$. Specific five-year survival was $60 \%$. Decreased specific survival was associated with: age $>40$ years; stages III or IV; location on the tongue, floor of the mouth, or base of the tongue; not performing surgical treatment, performing only chemotherapy or radiotherapy and chemotherapy; and residence in certain States of Brazil. The results emphasize the need to include assessment of regional disparities as a possibility for increasing health interventions and improving survival.
\end{abstract}

Mouth Neoplasms; Radiotherapy; Drug Therapy; Survivorship; Unified Health System
Gisele Macedo da Silva Bonfante 1

Carla Jorge Machado 1

Paulo Eduardo Alencar de Souza 2

Eli Iola Gurgel Andrade 1

Francisco de Assis Acurcio 1

Mariângela Leal Cherchiglia 1

\section{Resumo}

O objetivo foi analisar a sobrevida específica de cinco anos e fatores associados para câncer de boca no Brasil. Trata-se de coorte retrospectiva, cuja fonte de dados foi a Base Onco, que realizou o relacionamento probabilístico-determinístico de todos os registros de autorização para radioterapia elou quimioterapia pelo Sistema Único de Saúde, entre 2000 e 2006, gerando-se cadastro único para cada paciente. O presente estudo incluiu os pacientes diagnosticados entre 2002 e 2003 com câncer de boca, exceto lábio, e idade entre 19 e 100 anos $(N=6.180)$. A taxa de sobrevida específica em cinco anos foi de $60 \%$. Foram associados à menor sobrevida específica: ter idade > 40 anos; apresentar estádio III ou IV; localização em língua, assoalho de boca e base de língua; não realizar tratamento cirúrgico, realizar somente quimioterapia ou radioterapia e quimioterapia e residir em determinados estados do Brasil. Os resultados reforçam a necessidade de incluir a avaliação das disparidades dos territórios de planejamento como possibilidade para incrementar as ações de saúde e melhorar os indicadores de sobrevida.

Neoplasias Bucais; Radioterapia;

Quimioterapia; Sobrevida; Sistema Único de Saúde; 


\section{Introdução}

Nas últimas décadas, tem se verificado a progressiva ascensão das doenças crônico-degenerativas, dentre elas, o câncer. Segundo estimativas do Instituto Nacional de Câncer (INCA) 1, para o biênio 2012/2013, eram esperados, anualmente, 518 mil novos casos de câncer no Brasil.

Para o mesmo período, são estimados 14.170 novos casos anuais de câncer de boca, sendo 9.990 casos novos em homens e $4.180 \mathrm{em} \mathrm{mu-}$ lheres, correspondentes a um risco estimado de 10 casos novos a cada 100 mil homens e quatro a cada 100 mil mulheres. Esse câncer será o oitavo câncer mais incidente dentre todos os cânceres. Os estados de São Paulo, Rio de Janeiro e Minas Gerais respondem, no conjunto, por $56 \%$ da estimativa de incidência dentre todos os casos no país 1 .

O tipo histológico de câncer de boca mais frequente é o carcinoma de células escamosas 2,3 . Os principais fatores de risco são tabagismo e etilismo que apresentam efeito sinérgico e dose dependente $2,4,5$. Dessa forma, sua prevenção consiste basicamente em programas e medidas de controle ao consumo de tabaco e álcool, que, na verdade, visam à redução de diversas enfermidades. O diagnóstico precoce é o meio mais eficaz de que se dispõe para melhorar o prognóstico do câncer e consequentemente aumentar a taxa de sobrevida. Vale ressaltar, ainda, que, sendo diagnosticado tardiamente, o tratamento provavelmente envolverá cirurgias agressivas 4,6,7.

A despeito dessas considerações e de que um dos mais importantes objetivos de um sistema de saúde é prover diagnóstico precoce e assistência oportuna a sua população, mais da metade dos casos tem sido diagnosticada em estágios avançados, o que implica em pior prognóstico e diminuição da taxa de sobrevida 1,2,3,7,8,9,10,11,12. Sugerem-se diversas situações que poderiam levar a essa demora: profissionais que não sabem orientar corretamente pacientes; pacientes que não procuram o profissional por medo do diagnóstico ou somente o procuram quando já sofrem alguma restrição alimentar, de fala ou de convívio social 7,10,13

Por isso, este estudo tem o propósito de descrever e analisar a sobrevida específica de cinco anos para câncer de boca em nível nacional e seus fatores associados, uma vez que nenhum estudo anterior avaliou essas taxas no Brasil, nesse âmbito. Pretende-se ainda explorar a relação dessa sobrevida com os territórios de planejamento no país, no caso, tomando-se os estados brasileiros, uma vez que esses espaços, conforme definição da Portaria no 2.439/GM de 8 de dezembro de 2005 14, constituem a rede estadual ou re- gional de atenção oncológica e, assim, representam papel importante no atendimento a esses pacientes. Além disso, há evidências de que esses espaços sejam produtos e produtores de dinâmica social, tratando-se de territórios físicos, econômicos, políticos, sociais, culturais, epidemiológicos e de planejamento, cuja compreensão e análise do inter-relacionamento de seus próprios significados possibilitam melhor planejamento de assistência prestada em saúde 15.16.

\section{Metodologia}

Trata-se de coorte retrospectiva cuja fonte de dados foi o projeto intitulado Avaliação Econômico-epidemiológica do Tratamento Oncológico no Sistema Único de Saúde 2000-2006, do Grupo de Pesquisa em Economia da Saúde, que realizou o relacionamento determinístico-probabilístico de todos os registros de Autorização de Procedimentos de Alta Complexidade/Custo do Sistema de Informações Ambulatoriais do Sistema Único de Saúde (APAC-SIA/SUS) para radioterapia e/ ou quimioterapia, no período de 2000 a 2006, o que permitiu gerar um cadastro único para pacientes que realizaram tratamento oncológico ambulatorial no SUS, nesse período. Assim, todos os registros para um mesmo paciente estão sob um único registro. Informações oriundas do Sistema de Informações sobre Mortalidade (SIM) e Sistema de Internações Hospitalares (SIH) também foram pareadas a esses registros de forma que as internações, incluindo aquelas referentes a tratamentos cirúrgicos desses pacientes e a ocorrência de óbito, fossem agregadas às informações desses pacientes 17,18. As informações do SIH incluem os anos de 2000 a 2005, e as do SIM incluem os anos de 2003 a 2008. Assim foi composta a Base Onco, a qual permite a recomposição da trajetória de tratamento oncológico dos pacientes que foram diagnosticados com qualquer câncer e que receberam tratamento oncológico ambulatorial pelo SUS no período de 2000 a 2006. Esta investigação foi aprovada pelo Comitê de Ética em Pesquisa da Universidade Federal de Minas Gerais (ETIC 072/09).

Dos pacientes diagnosticados com câncer de boca, no período de 2000 a 2006, foram incluídos nesta análise, aqueles pacientes diagnosticados entre 1o de janeiro de 2002 e 31 de dezembro de 2003, exceto aqueles cuja localização anatômica era lábio, e que apresentavam idade entre 19 e 100 anos. Foram considerados todos os tipos de neoplasia maligna da boca - origem epitelial, mesenquimal e glandular. Entretanto, cerca de $90 \%$ a $95 \%$ de todos os tumores malignos de boca são do tipo carcinoma de células esca- 
mosas 2,19,20,21,22. A determinação deste período (2002/2003) se deve à necessidade do acompanhamento desses indivíduos pelo período mínimo de cinco anos. A exclusão do câncer de lábio se deve por ser uma neoplasia cujos fatores de risco, além do tabagismo e etilismo, envolvem principalmente a exposição solar e fenótipo relacionado à pigmentação da pele 2,23,24,25,26. Por último, menores de idade foram excluídos, pois o tratamento tende a ser diferenciado, inclusive quanto à autorização e ao pagamento dos procedimentos pelo SUS 27 .

A análise incluiu a descrição do perfil desses pacientes no Brasil. As sobrevidas global e específica foram calculadas a partir da data de diagnóstico do câncer de boca (confirmado por biópsia). Para a análise de sobrevida global, foi considerado "falha" o óbito por qualquer causa. As censuras incluíram pacientes que permaneceram vivos até o término do estudo. Para a análise de sobrevida específica, foi considerado "falha" o óbito cujo CID-10 foi câncer de boca (C00 a C06) 4 . Nesse caso, o paciente deveria ser relacionado ao CID-10 para câncer de boca em uma das linhas que descreviam a causa de óbito na Declaração de Óbito (DO): causa básica, linha A, linha B ou linha C. As censuras incluíram pacientes que permaneceram vivos após o término do estudo ou pacientes que foram a óbito por outras causas não relacionadas ao câncer de boca. As funções de sobrevida foram estimadas a partir do método univariado de Cox, segundo cada uma das variáveis explicativas do estudo, e os hazard ratios (HR) com os respectivos intervalos de 95\% de confiança (IC95\%) permitiram a seleção de variáveis que poderiam constituir o modelo múltiplo. O modelo de regressão de Cox foi utilizado para avaliação dos fatores associados à sobrevida, computando-se os HR com os respectivos IC95\%.

Além disso, diferenças entre os estados brasileiros podem representar um papel importante na avaliação de sobrevida desses pacientes. Assim, considerando que pacientes que iniciaram tratamento oncológico ambulatorial em um mesmo estado estariam expostos a um risco comum de ocorrência do evento de interesse, o modelo de fragilidade foi empregado. A utilização desse modelo reconhece a existência de correlação intragrupo, indicando que os pacientes assistidos, pelo menos, inicialmente em um mesmo estado tenham algum grau de semelhança no risco, decorrente de fatores associados às características assistenciais desses. Portanto, por meio da inclusão de um efeito aleatório (fragilidade) para o nível dos estados brasileiros (Unidade Federativa), esse modelo permitiu avaliar a associação entre os estados brasileiros e a sobrevida específica para câncer de boca, além de corrigir as estimativas dos efeitos das demais variáveis avaliadas. A força de associação para ambos os modelos de sobrevivência (sem e com fragilidade) foi demonstrada pelo HR e seu respectivo IC95\% 28.

Para comparação desses modelos, o teste de razão de verossimilhança foi utilizado, além da verificação do valor de significância da variável de fragilidade (Unidade Federativa de residência no primeiro tratamento oncológico ambulatorial).

As variáveis explicativas avaliadas foram sexo; idade ao diagnóstico; estadiamento clínico do tumor no momento do diagnóstico (biópsia); localização anatômica do câncer de boca; realização de algum tratamento cirúrgico para câncer de boca; combinação de tratamento oncológico ambulatorial (radioterapia e quimioterapia, associadas ou não); ocorrência de internações hospitalares, exceto aquelas que ocorreram para tratamento cirúrgico do câncer de boca. A variável de fragilidade foi "Unidade Federativa de residência no início do tratamento oncológico ambulatorial".

Quanto à localização anatômica do tumor, foram assim tratados, de acordo com o CID-10: lábio (C00, C00.0, C00.1, C00.2, C00.3, C00.4, C00.5, C00.6, C00.8, C00.9); base de língua (C01); língua (C02, C02.0, C02.1, C02.2, C02.3, C02.4, C02.8, C02.9); gengiva (C03, C03.0, C03.1, C03.9); assoalho bucal (C04, C04.0, C04.1, C04.8, C04.9); palato (C05, C05.0, C05.1, C05.2, C05.8, C05.9), outras partes e de partes não especificadas da boca (C06, C06.0, C06.1, C06.2, C06.8, C06.9).

Como variáveis respostas, foram utilizados o tempo entre o diagnóstico do câncer de boca e o óbito por câncer de boca e a ocorrência de óbito por câncer de boca, necessário para uma análise de sobrevida. As análises foram realizadas no software R 2.15.1 (The R Foundation for Statistical Computing, Viena, Áustria; http:// www.r-project.org).

\section{Resultados}

Do total de pacientes da Base Onco ( $\mathrm{N}=776.950)$, 22.249 pacientes foram diagnosticados com câncer de boca e iniciaram tratamento oncológico ambulatorial entre 2000 e 2006. Entre 2002 e 2003, 7.290 pacientes foram diagnosticados com esse câncer.

Desses, por representarem inconsistência de dados, apresentarem dados incompletos ou ausentes, foram excluídos: 198 pacientes por não possuírem registro de data de nascimento, 57 por incompatibilidade entre diagnóstico de câncer 
de boca e primeiro tratamento radioterápico ou quimioterápico autorizado, 498 por apresentarem entrada em tratamento oncológico anterior ao diagnóstico, oito por apresentarem óbito anterior ao diagnóstico e um por apresentar primeira metástase anterior ao diagnóstico. Restaram, portanto, 6.529 pacientes.

Então, segundo os critérios de inclusão estabelecidos para este estudo, foram eliminados os pacientes < 19 anos $(n=34)$ e $>100$ anos $(n=2)$ e aqueles cuja localização do câncer de boca foi lábio ( $\mathrm{n}=312$ ). Logo, esta investigação incluiu 6.180 pacientes diagnosticados entre 2002 e 2003 com câncer de boca, exceto câncer de lábio, em tratamento oncológico ambulatorial pelo SUS, disponíveis para a análise de sobrevida no Brasil.

Para a distribuição desses pacientes nos estados brasileiros, observou-se que residiam no início do tratamento oncológico ambulatorial: São Paulo (29\%); Minas Gerais (12\%), Rio Grande do Sul, Rio de Janeiro e Paraná (6\% a 7\% cada um); Bahia (5,12\%); Santa Catarina, Ceará e Piauí (4\% cada um); Paraíba (3,3\%); Espírito Santo, Rio Grande do Norte e Sergipe ( $2 \%$ a $3 \%$ cada um); Pará, Goiás, Alagoas e Mato Grosso do Sul (1\% a $2 \%$ cada um). O restante dos estados apresentou número inferior a $1 \%$.

Do total de pacientes analisados no Brasil, a maioria era do sexo masculino $(77,9 \%)$; com idade média de 59,0 \pm 13,1 anos; nascidos na Região Sudeste $(49,7 \%)$, Nordeste $(24,6 \%)$ e Sul $(18 \%)$; diagnosticados em estádios avançados (III: 34,5\% e IV: 41,1\%); com localização anatômica do tumor em língua $(30,7 \%)$ e assoalho bucal $(17,3 \%)$; que tiveram a radioterapia exclusiva como principal tratamento oncológico ambulatorial (57\%) e não tiveram internações não relacionadas ao tratamento cirúrgico do câncer de boca $(69,4 \%)$ (Tabela 1). Quanto à nacionalidade, $98 \%$ eram brasileiros. Para ano de diagnóstico, 51\% em 2002 e $49 \%$ em 2003.

Por sexo, a idade média para o feminino foi de $63,0 \pm 14,9$ anos, e, para o masculino, de 57,9 $\pm 12,3$ anos.

O tempo entre o diagnóstico do câncer de boca e a entrada em tratamento oncológico (cirúrgico, radioterápico e/ou quimioterápico) foi, em média, de aproximadamente três meses e apresentou mediana de $2 \pm 4,1$ meses. Quanto ao tempo médio de acompanhamento dos pacientes, esse foi de 29,4 meses (0-83 meses) e mediana de $16 \pm 27,2$ meses.

A média de internações por paciente, excluindo-se aquelas que ocorreram para tratamento cirúrgico do câncer de boca, foi de 0,64 \pm 1,33 internação por paciente. Restringindo-se essas internações somente àquelas cujo CID era de câncer de boca, mas não para tratamento ci- rúrgico desse, observou-se que $8,7 \%$ dos pacientes tiveram, pelo menos, uma internação relacionada ao câncer de boca, o que representa $29 \%$ de todas as causas de internações.

A sobrevida média específica para câncer de boca observada para o Brasil foi de 55,7 \pm 0,50 meses. A sobrevida específica em cinco anos foi de $60 \%$ (Figura 1a). Já a sobrevida global foi de $32,2 \pm 0,4$ meses, e a proporção, de 30\% (Figura $1 \mathrm{~b})$.

Adicionalmente, foi verificado que, dentre os pacientes que foram a óbito, excluindo-se aqueles que tiveram como causa de morte o câncer de boca, $38 \%$ morreram por cânceres localizados em orofaringe, faringe, laringe, nasofaringe, traqueia ou pulmão.

$\mathrm{Na}$ análise univariada, sete fatores estiveram positivamente associados à menor sobrevida específica para câncer de boca no Brasil com realização de radioterapia e/ou quimioterapia: sexo masculino; idade $\geq 40$ anos; apresentar estádio clínico do tumor III ou IV ao diagnóstico; localização anatômica do tumor em base de língua, língua e assoalho bucal; realizar tratamento oncológico ambulatorial que inclui quimioterapia no esquema terapêutico; ocorrência de internação, excluindo-se aquelas para tratamento cirúrgico do câncer de boca, e Unidade Federativa de residência no início do tratamento oncológico ambulatorial. Para estadiamento clínico e localização anatômica do tumor tomando-se os HR e seus respectivos IC95\%, os intervalos, para os estádios I e II, e a localização em gengiva ou palato se interpõem àqueles das categorias de referência (Tabela 2).

A Tabela 3 mostra os modelos de Cox sem e com fragilidade para a sobrevida específica de cinco anos no Brasil. A inclusão da fragilidade no modelo mostrou-se estatisticamente significante e acarretou aumento da estatística do teste de razão de verossimilhança de 302,2 para 407 .

Assim, permaneceram, no modelo final, as variáveis: idade, estadiamento clínico do tumor ao diagnóstico, localização anatômica do tumor, realização de tratamento cirúrgico para câncer de boca, combinação de tratamento oncológico ambulatorial, internação, exceto aquelas para tratamento cirúrgico do câncer de boca, e Unidade Federativa de residência no início do tratamento oncológico ambulatorial (Tabela 3).

Pacientes com idade $>40$ anos ao diagnóstico apresentaram risco de morrer $42 \%$ maior que pacientes com idade $\leq 40$ anos. Pacientes diagnosticados em estádios tardios mostraram risco aumentado em 47 e $74 \%$, respectivamente, comparados àqueles com carcinoma in situ. Aqueles cuja localização do tumor era em língua, base de língua ou assoalho bucal apresentaram aumento 
Tabela 1

Características dos pacientes diagnosticados com câncer de boca entre 2002 e 2003 e em tratamento oncológico ambulatorial pelo Sistema Único de Saúde, Brasil.

\begin{tabular}{|c|c|c|}
\hline Variáveis & $\mathrm{n}$ & $\%$ \\
\hline Total & 6.180 & 100,00 \\
\hline \multicolumn{3}{|l|}{ Sexo } \\
\hline Masculino & 4.812 & 77,90 \\
\hline Feminino & 1.368 & 22,10 \\
\hline \multicolumn{3}{|c|}{ Idade no momento do diagnóstico (anos) } \\
\hline $19-30$ & 77 & 1,25 \\
\hline $31-40$ & 287 & 4,64 \\
\hline $41-50$ & 1.364 & 22,07 \\
\hline $51-60$ & 1.791 & 28,98 \\
\hline+60 & 2.661 & 43,06 \\
\hline \multicolumn{3}{|l|}{ Localização anatômica do câncer } \\
\hline Língua & 1.899 & 30,73 \\
\hline Assoalho de boca & 1.066 & 17,25 \\
\hline Palato & 1.014 & 16,41 \\
\hline Outras partes da boca & 987 & 15,97 \\
\hline Base de língua & 963 & 15,58 \\
\hline Gengiva & 251 & 4,06 \\
\hline \multicolumn{3}{|c|}{ Região de residência no início do tratamento oncológico ambulatorial } \\
\hline Sudeste & 3.069 & 49,66 \\
\hline Nordeste & 1.522 & 24,63 \\
\hline Sul & 1.112 & 17,99 \\
\hline Centro-oeste & 277 & 4,48 \\
\hline Norte & 200 & 3,24 \\
\hline \multicolumn{3}{|c|}{ Estadiamento clínico no momento do diagnóstico } \\
\hline In situ & 157 & 2,54 \\
\hline I & 229 & 3,71 \\
\hline$\|$ & 1.124 & 18,19 \\
\hline III & 2.129 & 34,45 \\
\hline IV & 2.541 & 41,12 \\
\hline \multicolumn{3}{|c|}{ Combinação de tratamento oncológico ambulatorial } \\
\hline Radioterapia exclusiva & 3.571 & 57,00 \\
\hline Radioterapia e quimioterapia & 2.230 & 36,10 \\
\hline Quimioterapia exclusiva & 429 & 6,90 \\
\hline \multicolumn{3}{|c|}{ Realização de tratamento cirúrgico para câncer de boca } \\
\hline Não & 5.637 & 91,21 \\
\hline Sim & 543 & 8,79 \\
\hline \multicolumn{3}{|c|}{ Internação exceto aquelas para tratamento cirúrgico do câncer de boca } \\
\hline Não & 4.289 & 69,40 \\
\hline Sim & 1.891 & 30,60 \\
\hline
\end{tabular}

Fonte: Base Onco, 2000-2006. 
Figura 1

Sobrevida específica e global para pacientes diagnosticados com câncer de boca entre 2002 e 2003 e em tratamento oncológico no Sistema Único de Saúde, Brasil.

1a) Sobrevida específica

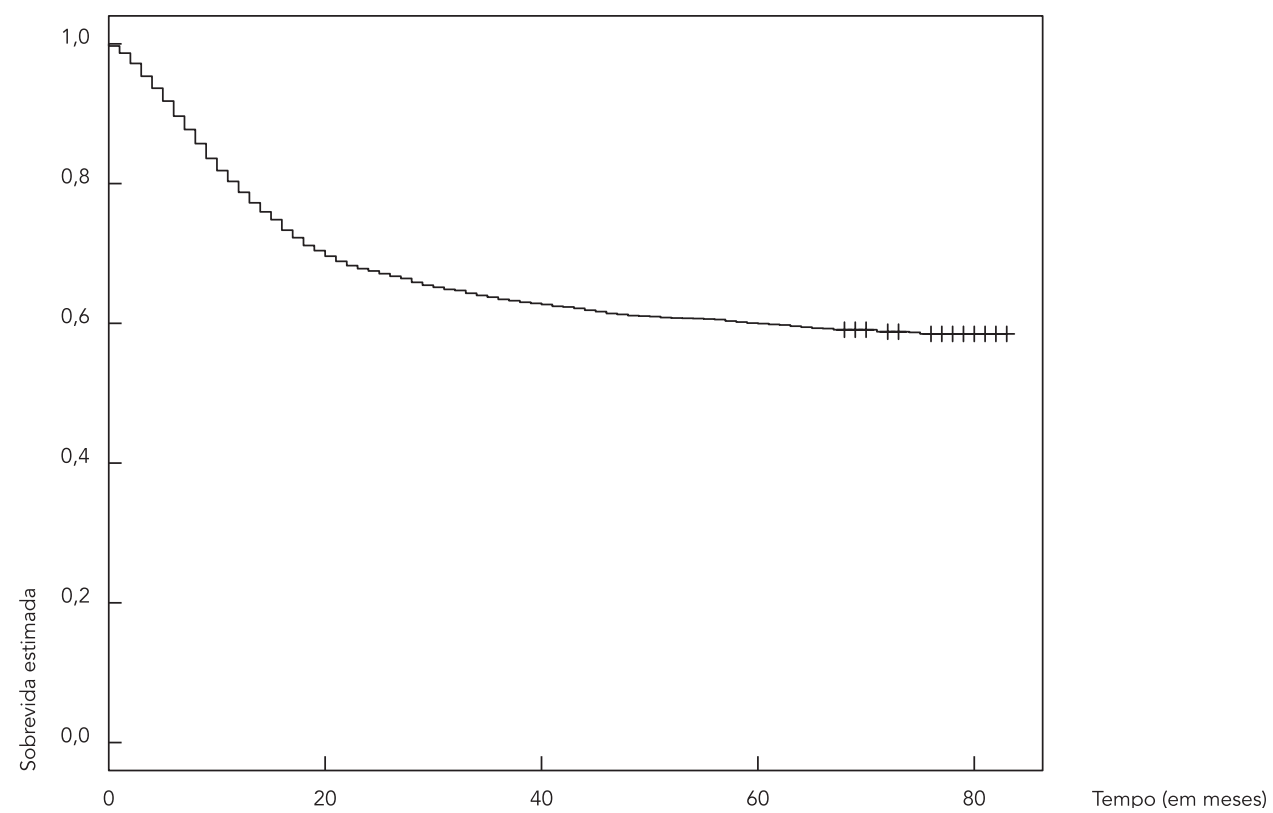

1b) Sobrevida global

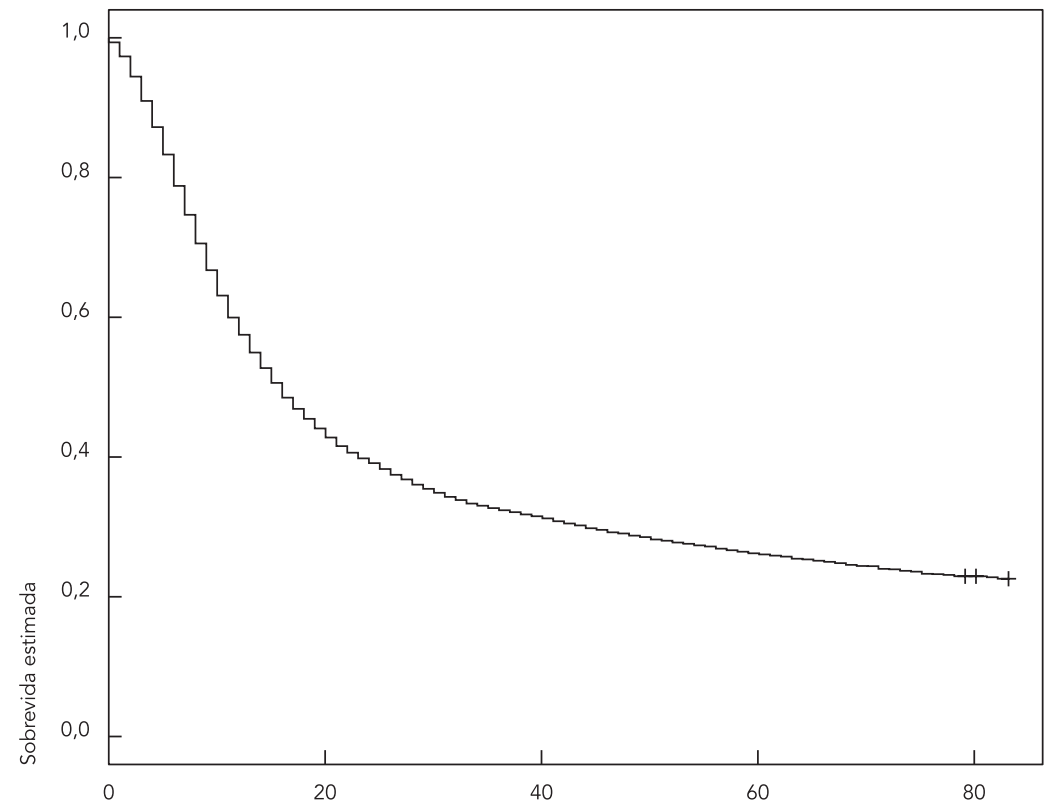

Tempo (em meses) 
Tabela 2

Modelo univariado de Cox para tempo até a ocorrência de óbito por câncer de boca em pacientes diagnosticados com câncer de boca entre 2002 e 2003 e em tratamento oncológico ambulatorial pelo Sistema Único de Saúde, Brasil.

\begin{tabular}{|c|c|c|c|}
\hline Variáveis & HR & IC95\% & Valor de $\mathrm{p}$ \\
\hline \multicolumn{4}{|l|}{ Sexo * } \\
\hline Masculino & 1,19 & $1,06-1,38$ & 0,002 \\
\hline \multicolumn{4}{|c|}{ Idade no momento do diagnóstico (anos) * } \\
\hline$>40$ & 1,41 & $1,15-1,73$ & 0,001 \\
\hline \multicolumn{4}{|c|}{ Estadiamento clínico no momento do diagnóstico * } \\
\hline I & 1,04 & $0,68-1,58$ & 0,851 \\
\hline ॥ & 0,96 & $0,67-1,37$ & 0,823 \\
\hline III & 1,53 & $1,87-2,16$ & 0,015 \\
\hline IV & 1,96 & $1,39-2,77$ & 0,000 \\
\hline \multicolumn{4}{|l|}{ Localização anatômica do tumor * } \\
\hline Base de língua & 1,45 & $1,23-1,72$ & 0,000 \\
\hline Língua & 1,58 & $1,37-1,83$ & 0,000 \\
\hline Assoalho de boca & 1,33 & $1,13-1,57$ & 0,010 \\
\hline Gengiva & 1,11 & $0,85-1,46$ & 0,438 \\
\hline Palato & 0,87 & $0,73-1,04$ & 0,134 \\
\hline \multicolumn{4}{|c|}{ Realização de alguma cirurgia relacionada ao câncer de boca * } \\
\hline Não & 1,16 & $1,00-1,35$ & 0,058 \\
\hline \multicolumn{4}{|c|}{ Combinação de tratamento oncológico ambulatorial * } \\
\hline Radioterapia e quimioterapia & 1,31 & $1,19-1,44$ & 0,000 \\
\hline Quimioterapia exclusiva & 2,13 & $1,79-2,53$ & 0,000 \\
\hline \multicolumn{4}{|c|}{ Internação exceto aquelas para tratamento cirúrgico do câncer de boca * } \\
\hline Sim & 1,35 & $1,23-1,49$ & 0,000 \\
\hline \multicolumn{4}{|l|}{ Ano de diagnóstico } \\
\hline 2003 & 0,98 & $0,90-1,07$ & 0,668 \\
\hline \multicolumn{4}{|c|}{ Unidade Federativa de residência no início do tratamento oncológico ambulatorial * } \\
\hline Rio de Janeiro & 1,66 & $1,40-1,96$ & 0,000 \\
\hline Minas Gerais & 0,83 & $0,71-0,99$ & 0,000 \\
\hline Espírito Santo & 1,62 & $1,24-2,11$ & 0,000 \\
\hline Rio Grande do Sul & 1,11 & $0,92-1,33$ & 0,298 \\
\hline Santa Catarina & 0,79 & $0,61-1,02$ & 0,072 \\
\hline Paraná & 1,28 & $1,07-1,53$ & 0,006 \\
\hline Mato Grosso do Sul & 1,15 & $0,75-1,78$ & 0,525 \\
\hline Mato Grosso & 1,10 & $0,70-1,74$ & 0,687 \\
\hline Goiás & 1,16 & $0,83-1,61$ & 0,378 \\
\hline Distrito Federal & 1,43 & $0,94-2,20$ & 0,980 \\
\hline Rondônia & 0,36 & $0,15-0,87$ & 0,024 \\
\hline Acre & 0,00 & $1,2665 \mathrm{E} 64$ & 0,910 \\
\hline Amazonas & 0,58 & $0,29-1,17$ & 0,127 \\
\hline Roraima & 0,00 & $5,601 \mathrm{E} 58$ & 0,902 \\
\hline Pará & 0,92 & $0,65-1,32$ & 0,657 \\
\hline Amapá & 0,00 & 1,721E14 & 0,960 \\
\hline Tocantins & 0,25 & $0,04-1,78$ & 0,167 \\
\hline Maranhão & 0,61 & $0,34-1,08$ & 0,087 \\
\hline Piauí & 1,24 & $0,79-1,97$ & 0,349 \\
\hline Ceará & 0,76 & $0,59-0,98$ & 0,034 \\
\hline Rio Grande do Norte & 0,98 & $0,71-1,36$ & 0,907 \\
\hline Paraíba & 0,62 & $0,45-0,86$ & 0,004 \\
\hline
\end{tabular}

(continua) 


\begin{tabular}{llll} 
Tabela 2 (continuação) & & & \\
\hline Variáveis & HR & IC95\% & Valor de $\mathbf{p}$ \\
\hline Unidade Federativa de residência no início do tratamento oncológico ambulatorial * & & & \\
$\quad$ Pernambuco & 1,06 & $0,84-1,33$ & 0,648 \\
Alagoas & 0,50 & $0,28-0,88$ & 0,017 \\
Sergipe & 0,58 & $0,41-0,83$ & 0,003 \\
Bahia & 1,23 & $1,00-1,51$ & 0,048 \\
\hline
\end{tabular}

Fonte: Base Onco, 2000-2006.

HR: hazard ratio; IC95\%: intervalo de $95 \%$ de confiança.

* Categorias de referência, respectivamente: feminino, $\leq 40$ anos, in situ, outras partes da boca, sim, radioterapia exclusiva, não, 2002, São Paulo.

Tabela 3

Modelos de Cox para tempo até a ocorrência de óbito por câncer de boca em pacientes diagnosticados com câncer de boca entre 2002 e 2003 e em tratamento oncológico ambulatorial pelo Sistema Único de Saúde, Brasil.

\begin{tabular}{|c|c|c|c|c|c|c|}
\hline \multirow[t]{2}{*}{ Variáveis explicativas } & \multicolumn{3}{|c|}{ Sem fragilidade } & \multicolumn{3}{|c|}{ Fragilidade } \\
\hline & HR & IC95\% & $\begin{array}{l}\text { Valor } \\
\text { de } p\end{array}$ & $\mathrm{HR}$ & IC95\% & $\begin{array}{l}\text { Valor } \\
\text { de } p\end{array}$ \\
\hline \multicolumn{7}{|l|}{ Individuais } \\
\hline \multicolumn{7}{|l|}{ Idade (anos) * } \\
\hline$>40$ & 1,42 & $1,16-1,75$ & 0,000 & 1,42 & $1,15-1,74$ & 0,001 \\
\hline \multicolumn{7}{|l|}{ Estadiamento clínico no momento do diagnóstico * } \\
\hline I & 1,10 & $0,72-1,68$ & 0,650 & 1,05 & $0,69-1,60$ & 0,820 \\
\hline ॥ & 1,00 & $0,70-1,44$ & 0,987 & 1,00 & $0,69-1,43$ & 0,980 \\
\hline III & 1,50 & $1,06-2,12$ & 0,021 & 1,47 & $1,04-2,09$ & 0,030 \\
\hline IV & 1,83 & $1,30-2,59$ & 0,000 & 1,74 & $1,23-2,46$ & 0,001 \\
\hline \multicolumn{7}{|l|}{ Localização anatômica do tumor * } \\
\hline Base de língua & 1,38 & $1,17-1,64$ & 0,000 & 1,35 & $1,14-1,60$ & 0,000 \\
\hline Língua & 1,60 & $1,39-1,86$ & 0,000 & 1,60 & $1,38-1,86$ & 0,000 \\
\hline Gengiva & 1,07 & $0,82-1,40$ & 0,633 & 1,13 & $0,86-1,48$ & 0,390 \\
\hline Assoalho de boca & 1,35 & $1,15-1,60$ & 0,000 & 1,29 & $1,10-1,53$ & 0,002 \\
\hline Palato & 0,93 & $0,77-1,11$ & 0,421 & 0,92 & $0,77-1,10$ & 0,370 \\
\hline \multicolumn{7}{|l|}{ Realização de tratamento cirúrgico para câncer de boca * } \\
\hline Não & 1,20 & $1,02-1,40$ & 0,023 & 1,29 & $1,10-1,51$ & 0,001 \\
\hline \multicolumn{7}{|l|}{ Combinação de tratamento oncológico ambulatorial * } \\
\hline Radioterapia e quimioterapia & 1,12 & $1,02-1,24$ & 0,024 & 1,18 & $1,066-1,31$ & 0,001 \\
\hline Somente quimioterapia & 1,75 & $1,47-2,09$ & 0,000 & 1,78 & $1,49-2,13$ & 0,000 \\
\hline \multicolumn{7}{|l|}{ Internação exceto aquelas para tratamento cirúrgico do câncer de boca * } \\
\hline Sim & 1,33 & $1,20-1,46$ & 0,000 & 1,27 & $1,15-1,41$ & 0,000 \\
\hline \multicolumn{7}{|l|}{ Variável de fragilidade } \\
\hline Unidade Federativa de residência no início do tratamento oncológico ambulatorial & & & & & & 0,000 \\
\hline Teste de razão de verossimilhança & 302,2 & & & 407 & & \\
\hline Variância da fragilidade & - & & & 0,105 & & \\
\hline
\end{tabular}

Fonte: Base Onco, 2000 a 2006.

HR: hazard ratio; IC95\%: intervalo de $95 \%$ de confiança.

* Categorias de referência, respectivamente: $\leq 40$ anos, in situ, outras partes da boca, sim, radioterapia exclusiva, não. 
do risco para ocorrência do óbito em relação à localização do tumor em outras partes e em partes não especificadas da boca. Aqueles que não foram submetidos a tratamento cirúrgico apresentaram risco maior de morrer em $30 \%$ em relação aos que o realizaram, bem como aqueles cujo tipo de tratamento oncológico ambulatorial foi quimioterapia exclusiva ou radioterapia associada à quimioterapia em relação ao tratamento radioterápico exclusivo. E pacientes que foram internados por qualquer causa, excluindo-se as internações para tratamento cirúrgico do câncer de boca, apresentaram risco aumentado de morrer em $30 \%$ em relação àqueles que não foram internados.

A Unidade Federativa de residência no início do tratamento oncológico ambulatorial se mostrou associada à sobrevida específica para câncer de boca em cinco anos. Observou-se que a variância da fragilidade $(0,105)$ foi significativa, indicando variabilidade na probabilidade de ocorrência do evento entre estados brasileiros. A Figura 2 mostra a estimativa pontual de fragilidade e seu IC95\% para cada um dos estados brasileiros, podendo ser classificada em três grupos: fragilidade $<1$, fragilidade média e fragilidade $>1$. Unidades Federativas com fragilidade $>1$ tendem a apresentar maior risco de morrer por câncer de boca, enquanto aquelas que apresentam valor $<1$ tendem a apresentar melhores taxas de sobrevida.

\section{Discussão}

Este estudo permitiu o conhecimento do perfil dos pacientes diagnosticados com câncer de boca, assistidos pelo SUS, cujo tratamento oncológico incluiu radioterapia e/ou quimioterapia. De forma geral, observou-se semelhança entre as características observadas e aquelas descritas por outros autores: maioria do sexo

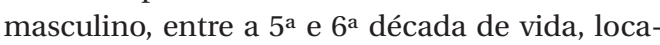
lizações mais frequentes para língua e assoalho bucal, além de diagnóstico em estádios tardios para a maior parte dos pacientes investigados 2,3,11,19,20,21,22,29,30,31.

A média de tempo entre diagnóstico e a entrada em tratamento foi de três meses. Segundo investigação realizada em hospital público em Belo Horizonte (Minas Gerais), em pacientes com carcinoma de células escamosas de cavidade oral confirmado por biópsia, o tempo entre o início dos primeiros sintomas e o tratamento, o qual correspondeu às somas dos tempos entre primeiros sintomas e consulta no hospital e tempo entre essa consulta e tratamento cirúrgico e/ ou radioterápico, foi de 143 dias para homens e de 28 dias para mulheres, sendo essa diferença estatisticamente significante 10 . Nesse caso, segundo autores, esse tempo seria influenciado pelo paciente, quando o tempo se concentra entre os sintomas e a consulta, e influenciado pelos profissionais, quando o tempo se concentra entre consulta e referência ao serviço de cirurgia de cabeça e pescoço no hospital e desse para o primeiro tratamento. Assim, como em nosso cálculo, a média de tempo aponta para uma incapacidade do sistema público brasileiro em prover tratamento oportuno ${ }^{8}$. Reforçam esses achados o tempo médio entre o diagnóstico e o início do tratamento detectado em Honorato et al. 9, que foi de 2,6 meses para pacientes diagnosticados com carcinoma de células escamosas de boca no INCA.

Foi possível estimar a sobrevida específica de cinco anos para câncer de boca (56 meses) e a sobrevida global de cinco anos (32 meses), bem como a taxa de sobrevida específica em cinco anos (60\%) e a taxa de sobrevida global em cinco anos (30\%). Esse último dado assemelha-se ao encontrado por Montoro et al. 32 que encontraram sobrevida global de $39 \%$ para pacientes diagnosticados com carcinoma de células escamosas de boca e tratados inicialmente por cirurgia com finalidade curativa. Esses autores indicaram que a baixa sobrevida estaria associada ao alto número de pacientes com metástases em pescoço e com localização do tumor em língua e assoalho bucal.

Também em relação à sobrevida, o estudo de Capilla et al. 33, realizado na Universidade de Granada (Granada, Espanha), verificou sobrevida global média de $2088 \pm 98$ dias para pacientes diagnosticados com carcinoma de células escamosas bucal (70 meses). Choi et al. 34 avaliaram o prognóstico do carcinoma de células escamosas em gengiva após procedimentos invasivos em pacientes diagnosticados e tratados em uma universidade na Coreia do Sul e encontraram sobrevida global em 5 anos de aproximadamente $61 \%$ e média de 98 meses. Hessel et al. 35 verificaram sobrevida global em 5 anos de $68,6 \%$ para pacientes com carcinoma de células escamosas em língua, diagnosticados em estágio T1-2, N0-1 e tratados na Universidade do Texas (Houston, Estados Unidos). Em relação à presente investigação, a taxa de sobrevida global encontrada nesses estudos dobra, achado esse possivelmente relacionado aos fatores de risco e causas de morte relacionadas a cada região geográfica e ao tipo de paciente investigado. Esperava-se, conforme observado, que a sobrevida global encontrada em nosso estudo fosse mais baixa, uma vez que envolveu pacientes submetidos à radioterapia e/ou à quimioterapia - além da cirurgia, em alguns casos. Para cânceres de boca 
Figura 2

Estimativa pontual da fragilidade e seu respectivo intervalo de 95\% de confiança (IC95\%) para cada uma das Unidades Federativas - sobrevida específica para câncer de boca, Brasil.

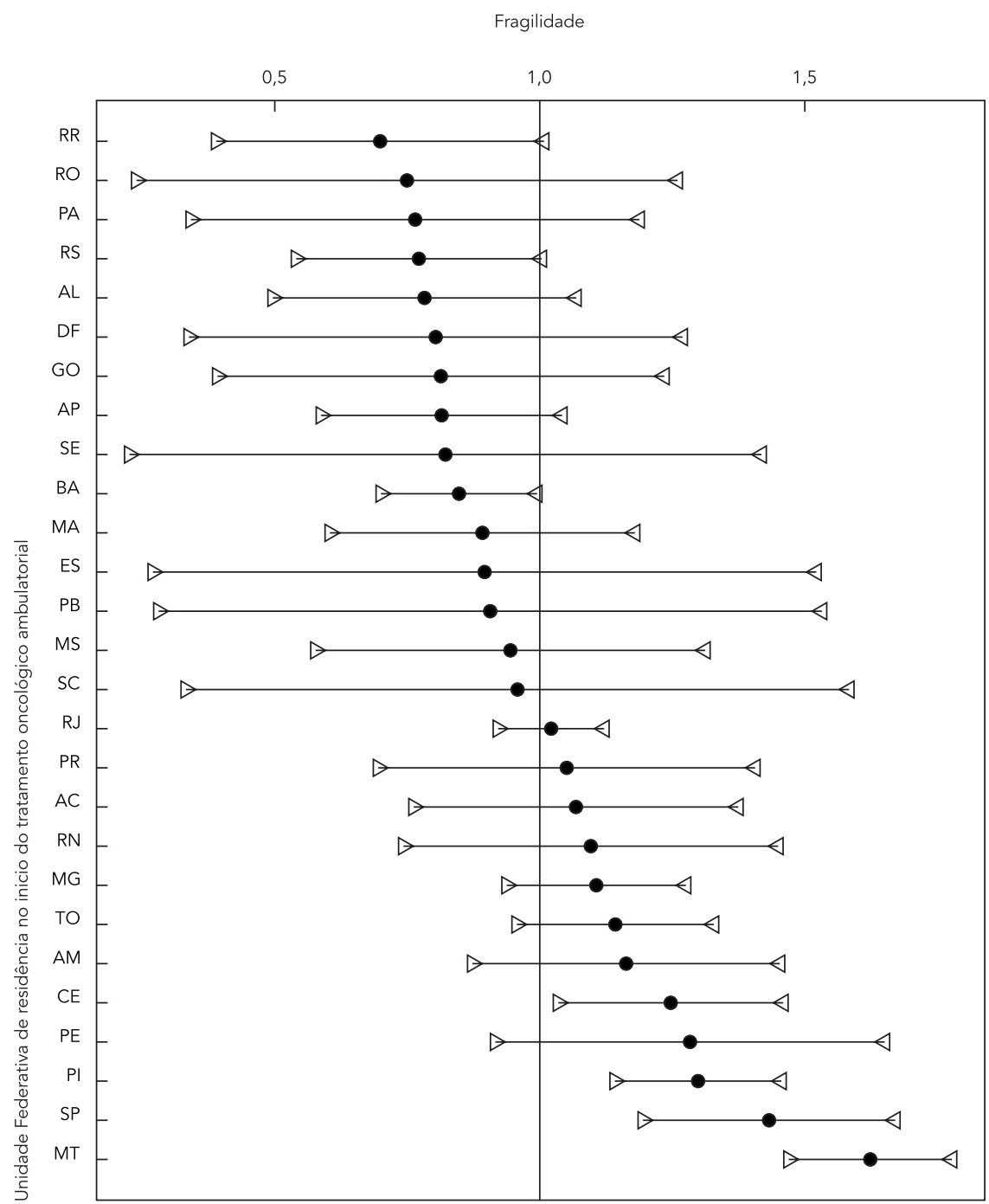

AC: Acre; AL: Alagoas; AM: Amazonas; AP: Amapá; BA: Bahia; CE: Ceará; DF: Distrito Federal; ES: Espírito Santo; GO: Goiás; MA: Maranhão; MG: Minas Gerais; MS: Mato Grosso do Sul; MT: Mato Grosso; PA: Pará; PB: Paraíba; PE: Pernambuco; PI: Piauí; PR: Paraná; RJ: Rio de Janeiro; RN: Rio Grande do Norte; RO: Rondônia; RR: Roraima; RS: Rio Grande do Sul; SC: Santa Catarina; SE: Sergipe; SP: São Paulo; TO: Tocantins. 
localmente avançados, o tratamento pode combinar cirurgia, radioterapia e quimioterapia de várias maneiras. A cirurgia é a primeira modalidade de tratamento em estágio precoce. A cirurgia apresenta alta previsibilidade, mas tem sua indicação afetada pela idade do paciente, condições médicas gerais, história anterior da doença e extensão da lesão, podendo comprometer o paciente fisicamente e esteticamente. A radioterapia poderá ser utilizada pré-cirurgia para tratamento de tumores de maiores dimensões, cuja intenção é reduzir o volume tumoral, ou como adjuvante para eliminação de células residuais de tumores invasivos removidos cirurgicamente. A quimioterapia é comumente administrada em cânceres de boca refratários, lesões extensas ou inoperáveis, a despeito de algum incremento na sobrevida livre de doença 36,37 . O tempo médio de seguimento curto observado em nossa investigação vai ao encontro dessa discussão.

Outros estudos avaliaram sobrevida global para câncer de boca. Brandizzi et al. 3 avaliaram 274 casos na Universidade de Buenos Aires (Buenos Aires, Argentina) e detectaram taxa de 39\% em cinco anos. Em Honorato et al. ${ }^{9}$, dos pacientes diagnosticados com carcinoma de células escamosas bucal no INCA, 57\% evoluíram para óbito. No estudo de Oliveira et al. ${ }^{25}$, que analisou pacientes diagnosticados com carcinoma de células escamosas bucal por biópsia na Faculdade de Medicina de Ribeirão Preto da Universidade de São Paulo, encontrou-se sobrevida de $24 \%$.

Em relação à sobrevida específica, o estudo de Chu et al. 38 envolveu pacientes com cânceres de cabeça e pescoço identificados no Registro de Câncer da Califórnia (Estados Unidos) e investigou a associação da sobrevida específica com o estado socioeconômico, controlando-se por outras variáveis individuais. Para o câncer oral, verificou-se taxa de sobrevida específica em cinco anos de $65 \%$ para aqueles com status socioeconômico mais baixo e de $75 \%$ para aqueles com status mais alto. A taxa de sobrevida específica de cinco anos em nosso estudo, portanto, assemelha-se àquela encontrada para pacientes com menor status socioeconômico.

Ao investigarmos as causas de óbito, que não o de câncer de boca, verificou-se maior ocorrência para outros cânceres de cabeça, pescoço e pulmão, tumores esses frequentemente associados ao tabagismo 38,39 . Isso reflete a teoria de campo de cancerização, sugerida por Slaughter et al. ${ }^{40}$, que afirma que várias partes das mucosas em contato com os produtos derivados do tabaco, principal fator etiológico para cânceres epiteliais de mucosa respiratória e digestiva superior, encontram-se afetadas. Assim, além das áreas clinicamente alteradas por lesões displá- sicas ou neoplásicas, várias outras células, em diferentes partes das mucosas expostas aos carcinógenos, já apresentam mutações no material genético, tornando-as mais susceptíveis a adquirir fenótipo neoplásico, constituindo-se condição cancerizável 40 .

Entre as variáveis associadas ao risco de morte por câncer de boca no Brasil, encontrou-se o aumento da idade, conforme outros estudos 25,38,39. Sugere-se que a diminuição da sobrevida com o aumento da idade esteja associada à maior incidência de doenças debilitantes, às maiores sequelas pós-tratamento e às complicações associadas ao envelhecimento 25 .

Neste estudo, ter sido diagnosticado nos estádios III e IV representou ter pior sobrevida específica, em relação ao carcinoma in situ. Nesse caso, sabe-se que o diagnóstico precoce é a medida mais eficaz para o aumento da sobrevida. Diagnósticos tardios, especialmente para carcinoma de células escamosas, evoluem para um tratamento geralmente mutilador, com cirurgias agressivas e tratamentos oncológicos mais complexos e de prognóstico duvidoso 36,37 . Pacientes com estadiamento clínico avançado apresentam prognóstico mais sombrio, como demonstrado por Brandizzi et al. ${ }^{3}$ que, após cinco anos do diagnóstico, detectaram taxas de sobrevida mais baixas: $34 \%$ para estágio III e $20 \%$ para o estágio IV do câncer bucal.

Oliveira et al. 25 encontraram piores taxas de sobrevida global em cinco anos para pacientes com recidivas e metástases de carcinoma epidermoide oral.

No presente estudo, os estádios I e II não aparecem como associados à maior sobrevida, podendo esse fato estar relacionado à não inclusão de pacientes que realizaram tratamento cirúrgico exclusivo na Base Onco. A cirurgia exclusiva aparece comumente como tratamento de escolha nos estádios iniciais dessa neoplasia 7,41.

A localização anatômica do tumor também se mostrou significativamente associada à sobrevida específica de cinco anos para câncer de boca. A boca apresenta subsítios com especialização estrutural e funcional. Isso parece refletir-se nos aspectos de evolução da neoplasia e, consequentemente, na sobrevida 36,37,42. Dependendo da localização anatômica do tumor, o comportamento biológico e a definição do tratamento variam. Lesões em base de língua e assoalho bucal se encontram em áreas de rica vascularização sanguínea e drenagem linfática, o que pode facilitar a disseminação metastática locorregional ou a distância, piorando o prognóstico e determinando a necessidade de tratamento mais agressivo 41,42. A investigação de Brandizzi et al. 3 para pacientes diagnosticados 
com carcinoma de células escamosas bucal também mostrou pior prognóstico para tumores em assoalho bucal (19\%) e língua (27\%).

Na presente investigação, também foi verificada menor sobrevida para pacientes não submetidos a tratamento cirúrgico. Resultado semelhante foi encontrado em estudo de sobrevida de cinco anos que avaliou pacientes com carcinoma de células escamosas em língua (45,5\% de sobrevida contra $96,1 \%$ em pacientes tratados cirurgicamente) ${ }^{43}$. A cirurgia é o principal tratamento para o câncer de boca, de forma exclusiva, ou em combinação com radioterapia e/ou quimioterapia $2,5,12,36,37,44$.

Em relação à combinação de tratamentos oncológicos ambulatoriais, verificou-se menor sobrevida para pacientes cujos esquemas terapêuticos envolviam quimioterapia. A exposição das células tumorais aos agentes quimioterápicos culmina na ativação de apoptose das células tumorais, mas também compromete células da medula óssea mitoticamente ativas. Assim, sua eficácia é prejudicada pela perda de seletividade, pela margem terapêutica estreita e pelo desenvolvimento de resistência às drogas utilizadas. Não há um consenso sobre drogas e esquemas a serem utilizados. Dessa forma, um regime que associe altas taxas de resposta completa, segurança de administração e menores efeitos à qualidade de vida desse paciente ainda são necessários. Portanto, os dados sugerem que a toxidade e a dificuldade em obter resposta clínica inicial possam impedir um melhor prognóstico associado a essa modalidade 36,37 .

Dentre as variáveis individuais avaliadas, encontrou-se associação inversa entre ocorrência de internação e sobrevida. Pacientes com necessidade de internação provavelmente apresentam maior número de intercorrências, comorbidades e/ou condições clínicas piores, que demandam maior número de hospitalizações e atuam de forma negativa sobre o risco de morrer desses pacientes. Esse achado está de acordo com o estudo de Castro et al. 44 , em que se observou que o risco de readmissões hospitalares está associado a um maior risco de morrer ao final das internações.

Na presente análise, optou-se pelo modelo de fragilidade, o que, em termos puramente estatísticos, justificaria a escolha pelo modelo de fragilidade ${ }^{28}$. Além disso, a variação encontrada entre o risco de morrer por câncer de boca nos estados brasileiros confirma a importância de se incluir a avaliação desses espaços para a identificação de pontos de atuação que possam impactar os resultados em saúde. Os padrões de sobrevida específica observados apontam para contrastes que estariam reproduzindo desigualdades presentes nesses espaços sociais, remetendo-nos para além de uma mudança de postura profissional e engajamento individual dos envolvidos, mas torna imprescindível um planejamento em saúde focado na necessidade de aumentar o acesso ao cuidado odontológico e em prover maior informação em saúde 8,16,45.

Limitações inerentes à utilização de um banco de dados de origem administrativa devem ser consideradas: identificação de lacunas de informação clínica; dificuldades na codificação dos procedimentos; caráter de faturamento; cobertura assistencial e ausência de informações socioeconômicas, sobre cor da pele, escolaridade, tabagismo e/ou etilismo. Também, quanto à utilização da DO, como fonte para causa de óbito, devido às questões relacionadas a esse sistema de informação, deve-se estar atento à subnotificação, ao sub-registro, ao preenchimento inadequado de dados, ao percentual de causas mal definidas e a cobertura variável desse em nível nacional, o que poderia implicar em superestimação da sobrevida. Em relação à descrição da causa de óbito, a distinção entre morte pela doença, devido à toxicidade do tratamento ou devido à causa independente é, muitas vezes, difícil, particularmente para doenças crônicas. Também, o seguimento passivo do indivíduo, que assume que ele esteja vivo até que uma notificação de morte seja encontrada (DO), pode levar a superestimação da sobrevida porque os casos considerados "perdidos" estarão, na verdade, mortos 46. Além disso, não foi possível incluir pacientes cujo tratamento foi cirurgia exclusiva, uma vez que o pareamento com o SIH tenha sido realizado a partir dos pacientes que apresentavam algum tratamento oncológico ambulatorial para câncer de boca pelo SUS. E, considerando a disponibilidade de dados de todos os sistemas de informação utilizados, observa-se que nem todos tiveram dados para o mesmo período.

A avaliação da sobrevida realizada nesta investigação e o estudo dos fatores associados, não somente ligados a características individuais, mas também verificados entre os estados brasileiros, reforçam a necessidade de incluir a abordagem e a avaliação das disparidades desses territórios de planejamento como possibilidade de incrementar as ações de saúde voltadas a esses pacientes e consequentemente alcançar melhores resultados no cuidado prestado e, portanto, melhores níveis dos indicadores de sobrevida. 


\section{Resumen}

El objetivo fue analizar la supervivencia específica a cinco años y los factores asociados al cáncer oral en Brasil. Se trata de una cohorte retrospectiva cuya fuente de datos fue la "Base Onco", que reúne todos los registros de autorización para radioterapia y quimioterapia en el sistema de salud público en Brasil entre 2000 y 2006. Este estudio incluyó a pacientes diagnosticados con cáncer de la boca-exceptuando el de labio-entre 2002 y 2003 y una edad entre 19 y 100 años $(N=6.180)$. La tasa de supervivencia específica a los cinco años fue del 60\%. Se asociaron con una menor supervivencia específica factores como: edad > 40 años; estadio III o IV; la localización del tumor en la lengua, suelo de la boca y base de la lengua; no realizar una cirugía para el tratamiento del cáncer oral; realizar sólo quimioterapia o radioterapia y quimioterapia conjuntamente; además de residir en ciertos estados brasileños. La inclusión de este enfoque refuerza la necesidad de incluir la evaluación de las disparidades en estos territorios como una oportunidad para mejorar las acciones de salud.

Neoplasias de la Boca; Radioterapia; Quimioterapia; Sobrevida; Sistema Único de Salud

\section{Colaboradores}

G. M. S. Bonfante e M. L. Cherchiglia contribuíram com a concepção do estudo, elaboração, análise e interpretação dos dados, elaboração do artigo e aprovação da versão final. C. J. Machado, P. E. A. Souza, E. I. G. Andrade e F. A. Acurcio contribuíram com a análise e interpretação dos dados, revisão crítica do artigo e aprovação da versão final.

\section{Agradecimentos}

Os autores agradecem ao Grupo de Pesquisa em Economia da Saúde (GPES), ao CNPq (processos 409729/2006-0 e 479033/2010-2) e à FAPEMIG (processos EDT 3284/06 e CDS-PPM-00098-11).

\section{Referências}

1. Instituto Nacional de Câncer. Estimativa 2012: incidência de câncer no Brasil. Rio de Janeiro: Instituto Nacional de Câncer; 2011.

2. Brener S, Jeunon FA, Barbosa AA, Grandinetti HAM. Carcinoma de células escamosas bucal uma revisão de literatura entre o perfil do paciente, estadiamento clínico e tratamento proposto. Rev Bras Cancerol 2007; 53:63-9.

3. Brandizzi D, Gandolfo M, Velazco ML, Cabrini RL Lanfranchi HE. Clinical features and evolution of oral cancer: a study of 274 cases in Buenos Aires, Argentina. Med Oral Patol Oral Cir Bucal 2008; 13:E544-8.

4. Instituto Nacional de Câncer. Falando sobre o câncer de boca. Rio de Janeiro: Instituto Nacional de Câncer; 2002.

5. Instituto Nacional de Câncer. Prevenção e controle do câncer: normas e recomendações do INCA. Rev Bras Cancerol 2002; 48:317-32.
6. Sassi LM, Dissenha JL, Simette RL, Stramandinoli RT, Pedruzzi PAG, Zanferrari FL, et al. Prevenção em câncer bucal: 20 anos de campanha antitabaco no Estado do Paraná, Brasil. Rev Bras Cir Cabeça Pescoço 2010; 39:184-6.

7. Tucci R, Borges FT, Aburad A, Carvalhosa AA. Avaliação de 14 casos de carcinoma epidermoide de boca com diagnóstico tardio. RSBO 2010; 7:231-8.

8. Abdo EM, Garrocho AA, Aguiar MCF. Perfil do paciente portador de carcinoma epidermoide da cavidade bucal, em tratamento no Hospital Mário Penna em Belo Horizonte. Rev Bras Cancerol 2002; 48:357-62.

9. Honorato J, Camisasca DR, Dias FL, Faria PAS Lourenço SQC. Análise de sobrevida global em pacientes diagnosticados com carcinoma de células escamosas de boca no ano de 1999. Rev Bras Epidemiol 2009; 12:69-81. 
10. Santos LCO, Batista OM, Cangussu MCT. Characterization of oral cancer diagnostic delay in the state of Alagoas. Braz J Otorhinolaryngol 2010; 76:416-22.

11. Castro MAF, Nardi CEM, Dedivitis RA. Câncer de boca e orofaringe em centro de referência terciária. Rev Bras Cir Cabeça Pescoço 2010; 39:38-42.

12. Murthy V, Agarwal JP, Laskar G, Gupta T, Budrukkar A, Pai P, et al. Analysis of prognostic factors in 1180 patients with oral cavity primary cancer treated with definitive or adjuvant radiotherapy. J Cancer Res Ther 2010; 6:282-9.

13. Carter LM, Ogden GR. Oral cancer awareness of undergraduate medical and dental students. BMC Med Educ 2007; 7:1-8.

14. Ministério da Saúde. Portaria no 2.439/GM de 8 de dezembro de 2005. Institui a Política Nacional de Atenção Oncológica: Promoção, Prevenção, Diagnóstico, Tratamento, Reabilitação e Cuidados Paliativos, a ser implantada em todas as unidades federadas, respeitadas as competências das três esferas de gestão. Diário Oficial de União 2005; 9 dez.

15. Barcellos C, Bastos FI. Geoprocessamento, ambiente e saúde: uma união possível? Cad Saúde Pública 1996; 12:389-97.

16. Mendes EV, Teixeira CF, Unglert CVS, Grego C, Araujo EC, Villarosa FN, et al. Distrito Sanitário: o processo social de mudança das práticas de saúde do Sistema Único de Saúde. São Paulo: Editora Hucitec/Rio de Janeiro: ABRASCO; 1999.

17. Cherchiglia ML, Guerra Júnior AA, Andrade EIG, Machado CJ, Acurcio FA, Meira Júnior W, et al. A construção da base de dados nacional em Terapia Renal Substitutiva centrada no indivíduo: aplicação do método de linkage determinístico-probabilístico. Rev Bras Estud Popul 2007; 24:163-7.

18. Queiroz OV, Guerra Júnior AA, Machado CJ, Andrade EIG, Meira Júnior W, Acurcio FA, et al. A construção da Base Nacional de Dados em Terapia Renal Substitutiva (TRS) centrada no indivíduo: relacionamento dos registros de óbitos pelo subsistema de Autorização de Procedimentos de Alta Complexidade (Apac/SIA/SUS) e pelo Sistema de Informações sobre Mortalidade (SIM) - Brasil, 2000-2004. Epidemiol Serv Saúde 2009; 18:107-20.

19. Pereira LA, Sobrinho JA, Rapoport A, Dedivitis RA. Epidemiologia do câncer bucal em Barretos, São Paulo. Rev Bras Cir Cabeça Pescoço 2003; 31:35-9.

20. Durazzo MD, Araujo CEN, Brandão Neto JS, Potenza AS, Costa P, Takeda F, et al. Clinical and epidemiological features of oral cancer in a medical school teaching hospital from 1994 to 2002: increasing incidence in women, predominance of advanced local disease, and low incidence of neck metastases. Clinics 2005; 60:293-8.

21. Antunes AA, Antunes AP, Silva PV, Avelar RL, Santos TS. Câncer de língua: estudo retrospectivo de vinte anos. Rev Bras Cir Cabeça Pescoço 2007; 36:152-4.

22. Souza RM, Sakae TM, Guedes AL. Características clínico-epidemiológicas de pacientes portadores de carcinomas da cavidade oral e orofaringe em clínica privada no Sul do Brasil. ACM Arq Catarin Med 2008; 37:32-41.
23. López EPM, Moral RMM, Martinez-Garcia C, Zanneti R, Rosso S, Serrano S, et al. Lifestyles environmental and phenotypic factors associated with lip cancer: a case-control study in Southern Spain. Br J Cancer 2003; 88:1702-7.

24. Antunes AA, Antunes AP. Estudo retrospectivo e revisão de literatura dos tumores dos lábios: experiência de 28 anos. Rev Bras Cancerol 2004; 50:295-300.

25. Oliveira LR, Ribeiro-Silva A, Zucolato S. Perfil de incidência e da sobrevida de pacientes com carcinoma epidermoide oral em uma população brasileira. J Bras Patol Med Lab 2006; 42:385-92.

26. Lopes PSD, Santos MA. Carcinoma de células escamosas labial em paciente jovem. Rev HCPA \& Fac Med Univ Fed Rio Gd do Sul 2012; 32:82-6.

27. Instituto Nacional de Câncer. Sistema de Informações Ambulatoriais do Sistema Único de Saúde. Bases técnicas para autorização de procedimentos de alta complexidade. APAC oncologia. Brasília: Instituto Nacional de Câncer; 2003.

28. Carvalho MS, Andreozzi VL, Codeço CT, Barbosa MTS, Shimakura SE. Análise de sobrevida: teorias e aplicações em saúde. Rio de Janeiro: Editora Fiocruz; 2005.

29. Sousa FACG, Paradella TC, Rosa LEB, Faig-Leite H. Carcinoma epidermoide em mucosa bucal: um breve levantamento. Rev Fac Odontol Porto Alegre 2008; 49:5-7.

30. Losi-Guembarovski R, Menezes RP, Poliseli F, Chaves VN, Kuasne H, Leichsenring A, et al. Oral carcinoma epidemiology in Paraná State, Southern Brazil. Cad Saúde Pública 2009; 25:393-400.

31. Santos LCO, Cangussu MCT, Batista OM, Santos JP. Câncer bucal: amostra populacional do Estado de Alagoas em hospital de referência. Bras J Otorhinolaryngol 2009; 75:524-9.

32. Montoro JRMC, Hicz AH, Souza L, Livingstone D, Melo DH, Tiveron RC, et al. Prognostic factors in squamous cell carcinoma of the oral cavity. Rev Bras Otorrinolaringol 2008; 74:861-6.

33. Capilla MV, Olid MNR, Gaya MVO, Botella CR, Ruiz VB. Factors related to survival from oral cancer in an Andalusian population sample (Spain). Med Oral Patol Oral Cir Bucal 2007; 12:E518-23.

34. Choi EJ, Zhang X, Kim HJ, Nam W, Cha IH. Prognosis of gingival squamous cell carcinoma diagnosed after invasive procedures. Asian Pacific J Cancer Prev 2011; 12:2649-52.

35. Hessel AC, Moreno MA, Hanna EY, Roberts DB, Lewin JS, El-Naggar AK, et al. Compliance with quality assurance measures in patients treated for early oral tongue cancer. Cancer 2010; 15:3408-16.

36. Hino S, Hamakawa H, Miyamoto Y, Ryoke K, Sekine J, Sasaki A, et al. Effects of a concurrent chemotherapy with S-1 for locally advanced oral cancer. Oncol Lett 2011; 2:839-43.

37. Silva SD, Hier M, Mlynarek A, Kowalski LP, AlaouiJamali MA. Recurrent oral cancer: current emerging therapeutic approaches. Front Pharmacol 2012; 3:1-7. 
38. Chu KP, Shema S, Wu S, Gomez SL, Chang ET, Le QT. Head and neck cancer specific survival based on socioeconomic status in Asians and Pacific Islanders. Cancer 2011; 117:1935-45.

39. Chang SW, Kareem SA, Kallarakkal TG, Merican AFMA, Abraham MT, Zain RB. Feature selection methods for optimizing clinicopathologic input variables in oral cancer prognosis. Asian Pac J Cancer Prev 2011; 12:2659-64.

40. Slaughter DP, Southwick HW, Smejkal W. Field cancerization in oral stratified squamous epithelium Clinical implications of multicentric origin. Cancer 1953; 6:963-8.

41. Instituto Nacional de Câncer. Carcinoma epidermoide da cabeça e pescoço. Rev Bras Cancerol 2001; 47:361-76.

42. Almeida FCS, Cazal C, Nunes FD, Araújo ME, Dias RB, Silva DP. Fatores prognósticos no câncer de boca. Rev Bras Ciênc Saúde 2011; 15:471-8.
43. Rana M, Iqbal A, Warraich R, Ruecker M, Eckardt AM, Gellrich NC. Modern surgical management of tongue carcinoma: a clinical retrospective research over a 12 years old period. Head Neck Oncol 2011; 3:1-8.

44. Castro MSM, Carvalho MS, Travassos C. Factors associated with readmission to a general hospital in Brazil. Cad Saúde Pública 2005; 21:1186-200.

45. Oliveira JC, Curado MP, Martins E, Moreira MAR. Incidência, mortalidade e tendência do câncer de cavidade oral e orofaringe em Goiânia de 1988 a 2003. Rev Bras Cir Cabeça Pescoço 2007; 36:70-4.

46. Bustamante-Teixeira MA, Faerstein E, Latorre MR. Técnicas de análise de sobrevida. Cad Saúde Pública 2002; 18:579-94.

Recebido em 26/Dez/2012

Versão final reapresentada em 01/Jul/2013

Aprovado em 06/Nov/2013 